\title{
Efficient Reactive Controller Synthesis for a Fragment of Linear Temporal Logic
}

\author{
Eric M. Wolff, Ufuk Topcu, and Richard M. Murray
}

\begin{abstract}
Motivated by robotic motion planning, we develop a framework for control policy synthesis for both nondeterministic transition systems and Markov decision processes that are subject to temporal logic task specifications. We introduce a fragment of linear temporal logic that can be used to specify common motion planning tasks such as safe navigation, response to the environment, persistent coverage, and surveillance. This fragment is computationally efficient; the complexity of control policy synthesis is a doubly-exponential improvement over standard linear temporal logic for both nondeterministic transition systems and Markov decision processes. This improvement is possible because we compute directly on the original system, as opposed to the automata-based approach commonly used. We give simulation results for representative motion planning tasks and compare to generalized reactivity(1).
\end{abstract}

\section{INTRODUCTION}

As autonomous vehicles and robots are used more widely, it is important for users to be able to accurately and concisely specify tasks. Additionally, given a task and a system, one would like to automatically synthesize a control policy that guarantees that the system will complete the specified task. In this context, we consider the problem of control policy synthesis in the presence of an adversarial environment that behaves either non-deterministically or probabilistically.

A widely used task specification language is linear temporal logic (LTL). LTL allows one to reason about how system properties change over time, and thus specify a wide variety of tasks, such as safety (always avoid B), response (if A, then B), persistence (eventually always stay in A), and recurrence (infinitely often visit A). While LTL is a powerful language for specifying system properties, the complexity of synthesizing a control policy that satisfies an LTL formula is doubly-exponential in the formula length for both nondeterministic and probabilistic systems [1], [2].

Temporal logics have been used to specify desired behaviors for robots and hybrid systems for which controllers can then be automatically synthesized. A common approach is to abstract the original continuous system as a finite discrete system, such as a non-deterministic transition system or a Markov decision process (MDP). Sampling-based motion planning techniques can be used for nonlinear systems to create a deterministic transition system that approximates the system, for which a satisfying control policy can be computed [3], [4]. A framework for abstracting a linear

Eric M. Wolff and Richard M. Murray are with the Department of Control and Dynamical Systems, California Institute of Technology, Pasadena, CA. Ufuk Topcu is with the Department of Electrical and Systems Engineering, University of Pennsylvania, Philadelphia, PA. The corresponding author is ewolffecaltech.edu system as a discrete transition system and then constructing a control policy that guarantees that the original system satisfies an LTL specification is presented in [5]. Reactive control policies are synthesized for linear systems in the presence of a non-deterministic environment in [6], and a receding horizon framework is used in [7] to handle the resulting blow-up in system size. Finally, control policies are created for Markov decision processes that represent a robots with noisy actuators for both LTL [8] and PCTL [9].

Motivated by robot motion planning, we introduce a fragment of LTL that can be used to specify tasks such as safe navigation, response to the environment, persistent coverage, and surveillance. For this fragment, we create control policies in time polynomial in the size of the system by computing reachable sets directly on the original system (as opposed to on the product of the system and a property automaton). The underlying algorithms are quite simple and the approach scales well. Preliminary experiments indicate that it outperforms standard implementations of generalized reactivity(1) (GR(1)) [10] on some motion planning problems.

There has been much interest in determining fragments of LTL that are computationally efficient to reason about. Fragments of LTL that have exponential complexity for control policy synthesis were analyzed in [11]. In the context of timed automata, certain fragments of LTL have been used for efficient control policy synthesis [12]. The GR(1) fragment can express many tasks, and control policies can be synthesized in time polynomial in the size of the system [10]. This fragment is extended to generalized Rabin(1), which is the largest fragment of specifications for which control policy synthesis can be done efficiently [13].

Our main contribution is an expressive fragment of LTL for efficient control policy synthesis for non-deterministic transition systems and Markov decision processes. A unified approach for control policy synthesis is presented that covers representative tasks and modeling frameworks. The algorithms used are simple and do not require detailed understanding of automata theory. The fragment that we use is effectively a Rabin acceptance condition, which allows us to compute directly on the system.

\section{PRoblem Formulation}

In this section we give background and introduce the main problem. An atomic proposition is a statement that is either True or False. The cardinality of a set $X$ is denoted by $|X|$. 


\section{A. System model}

We use finite transition systems and MDPs (introduced in Section VI) to model the system behavior. In robotics, however, one is usually concerned with continuous systems. This gap is partially bridged by constructive procedures for abstracting relevant classes of continuous systems as finite transition systems [14], [15]. Additionally, sampling-based methods, such as rapidly-exploring random trees [16] and probabilistic roadmaps [17], build a finite transition system that approximates a continuous system [3], [4].

Definition 1. A (finite) non-deterministic transition system (NTS) is a tuple $\mathcal{T}=\left(S, A, R, s_{0}, A P, L\right)$ consisting of a finite set of states $S$, a finite set of actions $A$, a transition function $R: S \times A \rightarrow 2^{S}$, an initial state $s_{0} \in S$, a set of atomic propositions $A P$, and a labeling function $L: S \rightarrow 2^{A P}$.

Let $A(s)$ denote the set of available actions at state $s$. Denote the parents of the states in the set $S^{\prime} \subseteq S$ by $\operatorname{Parents}\left(S^{\prime}\right):=\left\{s \in S \mid \exists a \in A(s)\right.$ and $\left.R(s, a) \cap S^{\prime} \neq \varnothing\right\}$. The set Parents $\left(S^{\prime}\right)$ includes all states in $S$ that can (possibly) reach $S^{\prime}$ in a single transition.

We assume that the transition system is non-blocking, i.e., $|R(s, a)| \geq 1$ for each state $s \in S$ and action $a \in A(s)$.

A deterministic transition system (DTS) is a nondeterministic transition system where $|R(s, a)|=1$ for each state $s \in S$ and action $a \in A(s)$. A run $\sigma=s_{0} s_{1} s_{2} \ldots$ of the transition system is an infinite sequence of its states, where $s_{i} \in S$ is the state of the system at index $i$ (also denoted $\sigma_{i}$ ) and for each $i=0,1, \ldots$, there exists $a \in A\left(s_{i}\right)$ such that $s_{i+1} \in R\left(s_{i}, a\right)$. A word is an infinite sequence of labels $L(\sigma)=L\left(s_{0}\right) L\left(s_{1}\right) L\left(s_{2}\right) \ldots$ where $\sigma=s_{0} s_{1} s_{2} \ldots$ is a run.

A memoryless control policy for a non-deterministic transition system $\mathcal{T}$ is a map $\mu: S \rightarrow A$, where $\mu(s) \in A(s)$ for state $s \in S$. A finite-memory control policy is a map $\mu: S \times M \rightarrow A \times M$ where the finite set $M$ is called the memory and $\mu(s, m) \in A(s) \times M$ for state $s \in S$ and mode $m \in M$. A control policy selects an action deterministically.

Given a state $s \in S$ and action $a \in A(s)$, there may be multiple possible successor states in the set $R(s, a)$, i.e., $|R(s, a)|>1$. A single successor state $t \in R(s, a)$ is non-deterministically selected. We interpret this selection (or action) as an uncontrolled (adversarial) environment resolving the non-determinism. A different interpretation of the environment will be given for MDPs in Section VI.

The set of runs of $\mathcal{T}$ with initial state $s \in S$ induced by a control policy $\mu$ is denoted by $\mathcal{T}^{\mu}(s)$.

\section{B. Linear temporal logic}

We use a fragment of linear temporal logic (LTL) to concisely and unambiguously specify desired system behavior. We begin by defining LTL, from which our fragment will inherit syntax and semantics. A comprehensive treatment of LTL is given in [18].

Syntax: LTL includes: (a) a set of atomic propositions, (b) the propositional connectives: $\neg$ (negation) and $\wedge$ (conjunction), and (c) the temporal modal operators: $\bigcirc$ (next) and $\mathcal{U}$ (until). Other propositional connectives such as $\vee$ (disjunction) and $\Longrightarrow$ (implication) and other temporal operators such as $\diamond$ (eventually), $\square$ (always), $\square \diamond$ (infinitely often), and $\diamond \square$ (eventually forever) can be derived.

An LTL formula is defined inductively as follows: (1) any atomic proposition is an LTL formula, (2) given formulas $\varphi_{1}$ and $\varphi_{2}, \neg \varphi_{1}, \varphi_{1} \wedge \varphi_{2}, \bigcirc \varphi_{1}$, and $\varphi_{1} \mathcal{U} \varphi_{2}$ are LTL formulas.

Semantics: An LTL formula is interpreted over an infinite sequence of states. Given an infinite sequence of states $\sigma=s_{0} s_{1} s_{2} \ldots$ and a formula $\varphi$, the semantics are defined inductively as follows: (i) for atomic proposition $p, s_{i} \vDash p$ if and only if (iff) $p \in L\left(s_{i}\right)$; (ii) $s_{i} \vDash \neg \varphi$ iff $s_{i} \vDash \varphi$; (iii) $s_{i} \vDash \varphi \wedge \psi$ iff $s_{i} \vDash \varphi$ and $s_{i} \vDash \psi$; (iv) $s_{i} \vDash \bigcirc \varphi$ iff $s_{i+1} \vDash \varphi$; and (v) $s_{i} \vDash \varphi \mathcal{U} \psi$ iff $\exists j \geq i$ s.t. $s_{j} \vDash \psi$ $\forall k \in[i, j)$ and $s_{k} \vDash \varphi$.

A propositional formula $\psi$ is composed of only atomic propositions and propositional connectives. We denote the set of states where $\psi$ holds by $[[\psi]]$.

An infinite sequence of states $\sigma=s_{0} s_{1} s_{2} \ldots$ satisfies the LTL formula $\varphi$, denoted by $\sigma \vDash \varphi$, if $s_{0} \vDash \varphi$. The system $\mathcal{T}$ under control policy $\mu$ satisfies the LTL formula $\varphi$ at state $s \in S$, denoted $\mathcal{T}^{\mu}(s) \vDash \varphi$ if and only if $\sigma \vDash \varphi$ for all $\sigma \in \mathcal{T}^{\mu}(s)$. Given a system $\mathcal{T}$, state $s \in S$ is winning for $\varphi$ if there exists a control policy $\mu$ such that $\mathcal{T}^{\mu}(s) \vDash \varphi$. Let $W \subseteq S$ denote the set of winning states.

\section{Problem Statement}

We now formally state the main problem of the paper and give an overview of our solution approach.

Problem 1. Given a non-deterministic transition system $\mathcal{T}$ with initial state $s_{0}$ and an LTL formula $\varphi$, determine whether there exists a control policy $\mu$ such that $T^{\mu}\left(s_{0}\right) \vDash \varphi$. Return the control policy $\mu$ if it exists.

Problem 1 is intractable in general. Determining if there exists such a control policy takes time doubly-exponential in the length of $\varphi$ [2]. Thus, we consider a fragment of LTL for which polynomial time solutions to Problem 1 exist. We will introduce such a fragment in Section II-D and solve Problem 1 for formulas of this form. We begin by solving Problem 1 for the special case of a deterministic transition system in Section IV. While this discussion is subsumed by that for the non-deterministic transition system, it allows for stronger results. We solve Problem 1 for nondeterministic transition systems in Section V. Finally, we solve an analogous problem for MDPs in Section VI.

\section{A fragment of $L T L$}

We now introduce a fragment of LTL that can specify a wide range motion planning tasks such as safe navigation, immediate response to the environment, persistent coverage, and surveillance. We consider formulas of the form

$$
\varphi=\varphi_{\text {safe }} \wedge \varphi_{\text {act }} \wedge \varphi_{\text {per }} \wedge \varphi_{\text {rec }}
$$

where

$$
\begin{aligned}
\varphi_{\text {safe }}:=\square p_{1}, & \varphi_{\text {act }}:=\bigwedge_{j \in I_{2}} \square\left(p_{2, j} \Longrightarrow \bigcirc q_{2, j}\right), \\
\varphi_{\text {per }}:=\diamond \square p_{3}, \quad & \varphi_{\text {rec }}:=\bigwedge_{j \in I_{4}} \square \diamond p_{4, j}
\end{aligned}
$$


and $p_{1}:=\bigwedge_{j \in I_{1}} p_{1, j}$ and $p_{3}:=\bigwedge_{j \in I_{3}} p_{3, j}$ with $\bigwedge_{j \in I_{1}} \square p_{1, j}=$ $\square \bigwedge_{j \in I_{1}} p_{1, j}$ and $\bigwedge_{j \in I_{3}} \diamond \square p_{3, j}=\diamond \square \bigwedge_{j \in I_{3}} p_{3, j}$, respectively. In the above definitions, $I_{1}, \ldots, I_{4}$ are finite index sets and $p_{i, j}$ and $q_{i, j}$ are propositional formulas for any $i$ and $j$.

Remark 1. Guarantee and obligation, i.e., $\diamond p$ and $\square(p \Longrightarrow$ $\diamond q)$ respectively (where $p$ and $q$ are propositional formulas), are not included in (1). We show how to include these specifications in Section VIII-A. It is also natural to consider specifications that are disjunctions of formulas of the form (1). We give conditions for this extension in Section VIII-B.

Remark 2. The fragment in formula (1) is clearly a strict subset of LTL. This fragment is incomparable to other commonly used temporal logics, such as computational tree logic (CTL and PCTL), and GR(1). The fragment that we consider allows persistence $(\diamond \square)$ to be specified, which cannot be specified in either CTL or GR(1). However, it cannot express existential path quantification as in CTL or allow disjunctions of formulas as in GR(1) [10], [18]. The fragment is part of the generalized Rabin(1) logic [13] and the $\mu$-calculus of alternation depth two [19].

\section{PRELIMINARIES}

\section{A. Acceptance conditions}

We now give acceptance conditions from classical automata theory [20] for the LTL fragment introduced in Section II-D. These acceptance conditions are critical to the development in this paper, as much of the later analysis depends on them. Effectively, we reason about satisfaction of LTL formulas in terms of set operations between a run $\sigma$ of $\mathcal{T}=\left(S, A, R, s_{0}, A P, L\right)$ and subsets of $S$ where particular propositional formulas hold. We first define acceptance conditions for a run and then extend it to a system $\mathcal{T}$.

Definition 2. Let $\sigma$ be a run of the system $\mathcal{T}, \operatorname{Inf}(\sigma)$ denote the set of states that are visited infinitely often in $\sigma$, and $\operatorname{Vis}(\sigma)$ denote the set of states that are visited at least once in $\sigma$. Given propositional formulas $\varphi$ and $\psi$, relate satisfaction of an LTL formula with acceptance conditions as follows

- $\sigma \vDash \square \varphi$ iff $\operatorname{Vis}(\sigma) \subseteq[[\varphi]]$,

- $\sigma \vDash \diamond \square \varphi$ iff $\operatorname{Inf}(\sigma) \subseteq[\varphi \varphi]$,

- $\sigma \vDash \square \diamond \varphi$ iff $\operatorname{Inf}(\sigma) \cap[[\varphi]] \neq \varnothing$,

- $\sigma \vDash \square(\psi \Longrightarrow \bigcirc \varphi)$ iff $\sigma_{i} \in\left[[\psi]\right.$ or $\sigma_{i+1} \notin[[\varphi]]$ for all $i$.

A run satisfies a conjunction of LTL formulas if and only if it satisfies all corresponding acceptance conditions. Acceptance for a system $\mathcal{T}$ is extended over runs in the obvious manner.

In automata theory, $\square \diamond \varphi$ is called a Büchi acceptance condition and $\diamond \square \varphi$ is called a co-Büchi acceptance condition. The conjunction of both a Büchi and a co-Büchi acceptance condition is a Rabin acceptance condition with one pair [20].

An example is given in Figure 1. The non-deterministic transition system $\mathcal{T}$ has states $S=\{1,2,3,4\}$; labels $L(1)=$ $\{A\}, L(2)=\{C\}, L(3)=\{B\}, L(4)=\{B, C\}$; a single action called 0 ; and transitions $R(1,0)=\{2,3\}, R(2,0)=$ $\{2\}, R(3,0)=\{4\}, R(4,0)=\{4\}$. From the acceptance conditions, it follows that states $\{2,4\}$ are winning for

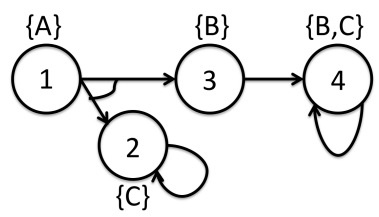

Fig. 1. Example of a non-deterministic transition system

formula $\square(A \vee C)$, states $\{2,3,4\}$ are winning for formula $\square(A \Longrightarrow \bigcirc B)$, states $\{1,2,3,4\}$ are winning for formula $\square \diamond C$, and states $\{3,4\}$ are winning for formula $\diamond \square B$. State 4 is winning for all of the formulas above.

\section{B. Graph Theory}

We will often consider a non-deterministic transition system as a graph with the natural bijection between the states and transitions of the transition system and the vertices and edges of the graph. Let $G=(S, R)$ be a directed graph (digraph) with vertices $S$ and edges $R$. Let there be an edge $e$ from vertex $s$ to vertex $t$ if and only if $t \in R(s, a)$ for some $a \in A(s)$. A walk $w$ is a finite edge sequence $w=e_{0} e_{1} \ldots e_{p}$. Denote the set of all nodes visited along walk $w$ by $\operatorname{Vis}(w)$.

A digraph $G=(S, R)$ is strongly connected if there exists a path between each pair of vertices $s, t \in S$ no matter how the non-determinism is resolved. A digraph $G^{\prime}=\left(S^{\prime}, R^{\prime}\right)$ is a subgraph of $G=(S, R)$ if $S^{\prime} \subseteq S$ and $R^{\prime} \subseteq R$. The subgraph of $G$ restricted to states $S^{\prime} \subseteq S$ is denoted by $\left.G\right|_{S^{\prime}}$. A digraph $G^{\prime} \subseteq G$ is a strongly connected component if it is a maximal strongly connected subgraph of $G$.

\section{Reachability}

We define controlled reachability in a non-deterministic transition system $\mathcal{T}$ with a value function. Let $B \subseteq S$ be a set of states that the controller wants the system to reach. Let the controlled value function for system $\mathcal{T}$ and target set $B$ be a map $V_{B, \mathcal{T}}^{c}: S \rightarrow \mathbb{N} \cup \infty$, whose value $V_{B, \mathcal{T}}^{c}(s)$ at state $s \in S$ is the minimum (over all possible control policies) number of transitions needed to reach the set $B$, given the worst-case resolution of the non-determinism. If the value $V_{B, \mathcal{T}}^{c}(s)=\infty$, then the non-determinism can prevent the system from reaching set $B$ from state $s \in S$. For example, consider the system in Figure 1 with $B=\{4\}$. Then, $V_{B}^{c}(1)=$ $\infty, V_{B}^{c}(2)=\infty, V_{B}^{c}(3)=1$, and $V_{B}^{c}(4)=0$.

The value function satisfies the optimality condition

$$
V_{B, \mathcal{T}}^{c}(s)=\min _{a \in A(s)} \max _{t \in R(s, a)} V_{B, \mathcal{T}}^{c}(t)+1,
$$

for all $s \in S$. Algorithm 1 computes the value function by backwards iteration from the target set $B$ in $O(|S|+|R|)$ time. At every iteration, a state is assigned a finite value if it can reach a state with a finite value in a single transition, no matter how the non-determinism is resolved.

An optimal control policy $\mu_{B}$ for reaching the set $B$ is implicitly encoded in a value function $V_{B, \mathcal{T}}^{c}$ that satisfies (2). Optimal control policies are memoryless for reachability [21]. Such a policy can be computed at each state $s \in S$ as

$$
\mu_{B}(s)=\underset{a \in A(s)}{\arg \min } \max _{t \in R(s, a)} V_{B, \mathcal{T}}^{c}(t)+1 .
$$




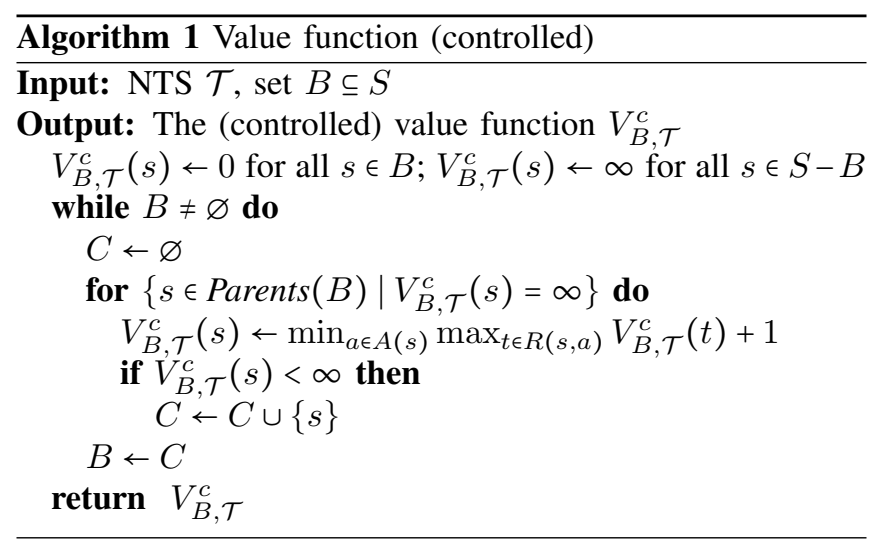

We use the value function to define the controllable predecessor set for a given system $\mathcal{T}$ with target set $B \subseteq S$. Let

$$
C \operatorname{Pr} e_{\mathcal{T}}^{\infty}(B):=\left\{s \in S \mid V_{B, \mathcal{T}}^{c}(s)<\infty\right\}
$$

be the set of all states that can reach a state in $B$ for any resolution of the non-determinism.

We define forced reachability similarly. Let the forced value function for system $\mathcal{T}$ and target set $B$ be a map $V_{B, \mathcal{T}}^{f}: S \rightarrow \mathbb{N} \cup \infty$, whose value $V_{B, \mathcal{T}}^{f}(s)$ at state $s \in S$ is the maximum (over all possible control policies) number of transitions before reaching the set $B$. The forced value function satisfies the optimality condition

$$
V_{B, \mathcal{T}}^{f}(s)=\max _{a \in A(s)} \max _{t \in R(s, a)} V_{B, \mathcal{T}}^{f}(t)+1 .
$$

For a given system $\mathcal{T}$ with target set $B \subseteq S$, the forced predecessor set

$$
\operatorname{FPre}_{\mathcal{T}}^{\infty}(B):=\left\{s \in S \mid V_{B, \mathcal{T}}^{f}(s)<\infty\right\},
$$

is the set of all states from which no control policy can avoid reaching a state in $B$.

Remark 3. We consider the case where the controller selects an action, and then the environment selects the next state. Our results easily extend to the case, used in GR(1) [10], where the environment first resolves the non-determinism (selects an action) and then the controller selects its action.

Remark 4. It is not necessary to compute the exact value function at each state when computing the predecessor sets, only whether it is finite or infinite.

\section{SOLUTION FOR DETERMINISTIC TRANSITION SYSTEMS}

We first create control policies for deterministic transition systems. We will compute the winning set $W \subseteq S$ for each specification separately and then combine them in Algorithm 2. Recall that $\mathcal{T}$ is originally non-blocking.

First, remove all actions from $\mathcal{T}$ that do not satisfy the next-step response specification $\varphi_{\text {act }}=\bigwedge_{j \in I_{2}} \square\left(p_{2, j} \Longrightarrow\right.$ $\left.\bigcirc q_{2, j}\right)$. For each $j \in I_{2}$, remove an action $a \in A(s)$ from a state $s \in S$ if $s \in \llbracket p_{2, j} \rrbracket$ and $R(s, a) \nsubseteq \llbracket \llbracket q_{2, j} \rrbracket$. Let $B \subseteq S$ contain all states that are blocking (due to the removal of an action). Create the subgraph $\mathcal{T}_{\text {act }}:=\left.\mathcal{T}\right|_{S-F \operatorname{Pre}} ^{\infty}(B)$.
Proposition 1. A state is in $\mathcal{T}_{\text {act }}$ if and only if it is winning for $\varphi_{\text {act }}$.

Proof: An action is removed from $\mathcal{T}$ if and only if it directly violates the acceptance condition for $\varphi_{\text {act }}$. All blocking states, i.e., those in $B \subseteq S$, must use an action that was removed. Thus, the set $F \operatorname{Pre} e_{\mathcal{T}}^{\infty}(B)$ contains all and only states that violate the acceptance condition for $\varphi_{\text {act }}$. $\mathcal{T}_{\text {act }}$ is non-blocking, so any run of the system satisfies $\varphi_{\text {act }}$.

Next, remove the states that violate the safety specification $\varphi_{\text {safe }}=\square p_{1}$ by creating the subgraph $\mathcal{T}_{\text {safe }}:=$ $\left.\mathcal{T}\right|_{S-F \operatorname{Pre}} ^{\infty}\left(S-\left[\left[p_{1}\right]\right) \cdot\right.$

Proposition 2. A state is in $\mathcal{T}_{\text {safe }}$ if and only if it is winning for $\varphi_{\text {safe }}$.

Proof: The acceptance condition for $\varphi_{\text {safe }}$ is $\operatorname{Vis}(\sigma) \subseteq$ $\left.\llbracket\left[p_{1}\right]\right]$. The set $\operatorname{FPre} e_{\mathcal{T}}^{\infty}\left(S-\left[\left[p_{1} \rrbracket\right)\right)\right.$ contains a state if and only if it either is not in $\left[\left[p_{1}\right]\right]$ and or cannot avoid visiting a state not in $\left[\left[p_{1}\right]\right]$. $\mathcal{T}_{\text {safe }}$ is non-blocking, so any run of the system satisfies $\varphi_{\text {safe }}$.

Incorporate the persistence specification $\varphi_{\text {per }}=\diamond \square p_{3}$ by creating the subgraph $\mathcal{T}_{\text {per }}:=\left.\mathcal{T}\right|_{S-F P r e}\left(S-\llbracket\left[p_{3} \rrbracket\right)\right.$. The winning set is $C \operatorname{Pre} e_{\mathcal{T}}^{\infty}\left(S_{\text {per }}\right)$, where $S_{\text {per }}$ is the set of states in $\mathcal{T}_{\text {per }}$.

Proposition 3. A state is in $\mathcal{T}_{\text {per }}$ if it is winning for $\varphi_{\text {per }}$.

Proof: As in Proposition 2, but with acceptance condition $\operatorname{Inf}(\sigma) \subseteq \llbracket\left[p_{3} \rrbracket\right.$.

We now compute the winning set for the recurrence specification $\varphi_{\text {rec }}=\bigwedge_{j \in I_{4}} \square \diamond p_{4, j}$ by computing the sets of states that can be visited infinitely often.

Proposition 4. Let $\sigma$ be a run of $\mathcal{T}$. If states $s, t \in \operatorname{Inf}(\sigma)$, then they must be in the same strongly connected component.

Proof: By definition of $\operatorname{Inf}(\sigma)$, states $s$ and $t$ are visited infinitely often. Thus, there must exist a walk starting at $s$ and ending at $t$ and vice versa. Thus, $s$ and $t$ are in the same strongly connected component.

The strongly connected components of $\mathcal{T}$ can be computed in $O(|S|+|R|)$ time using Tarjan's algorithm [22]. Let $S C C\left(\mathcal{T}_{\text {per }}\right)$ be the set of all strongly connected components of $\mathcal{T}_{\text {per }}$ that have at least one transition between states in the component. A strongly connected component $C \epsilon$ $S C C\left(\mathcal{T}_{\text {per }}\right)$ is accepting if $C \cap \llbracket\left[p_{4, j} \rrbracket \neq \varnothing\right.$ for all $j \in I_{4}$. Let $\mathcal{A}$ be the set of all accepting strongly connected components and $S_{\mathcal{A}}:=\{s \in S \mid s \in C$ for some $C \in \mathcal{A}\}$. Every state in an accepting strongly connected component is in the winning set $W:=\operatorname{CPre}_{\mathcal{T}}^{\infty}\left(S_{\mathcal{A}}\right)$.

Proposition 5. A state is in $S_{\mathcal{A}}$ if it is winning for $\varphi_{\text {rec }}$.

Proof: The relevant acceptance condition is $\operatorname{Inf}(\sigma) \cap$ $\llbracket p_{4, j} \rrbracket \neq \varnothing$ for all $j \in I_{4}$. By definition, every state in $C \in \mathcal{A}$ can be visited infinitely often. Since $C \cap\left[\left[p_{4, j}\right]\right] \neq \varnothing$ for all $j \in I_{4}$, the result follows.

We now give an overview of our approach for control policy synthesis for deterministic transition systems in Algorithm 2. Optionally, remove all states from $\mathcal{T}$ that cannot 
be reached from the initial state $s_{0}$ in $O(|S|+|R|)$ time using breadth-first search from $s_{0}$ [22]. Compute the set of states $W$ that are winning for $\varphi$ (lines 1-5). If the initial state $s_{0} \notin W$, then no control policy exists (lines 6-8). If $s_{0} \in W$, compute a walk on $\mathcal{T}_{\text {safe }}$ from $s_{0}$ to a state $t \in C$ for some accepting strongly connected component $C \in \mathcal{A}$ and where $t \in \cup_{j \in I_{4}}\left[\left[p_{4, j}\right]\right]$ (lines 9-10). Compute a walk $\sigma_{\text {suf }}$ starting and ending at state $t$ such that $\operatorname{Vis}\left(\sigma_{\text {suf }}\right) \cap\left[\left[p_{4, j}\right]\right] \neq \varnothing$ for all $j \in I_{4}$ and $\operatorname{Vis}\left(\sigma_{\text {suf }}\right) \subseteq C$ (line 11). The control policy is implicit in the (deterministic) run $\sigma=\sigma_{\text {pre }}\left(\sigma_{\text {suf }}\right)^{\omega}$, where $\omega$ denotes infinite repetition. The total complexity of the algorithm is $O\left(\left|I_{2}\right|(|S|+|R|)\right)$ to check feasibility and $O\left(\left(\left|I_{2}\right|+\left|I_{4}\right|\right)(|S|+|R|)\right)$ to compute a control policy, where the extra term is for computing $\sigma_{\text {suf. Note that any policy }}$ that visits every state in $C$ infinitely often (e.g., randomized or round-robin) could be used to avoid computing $\sigma_{\text {suf }}$.

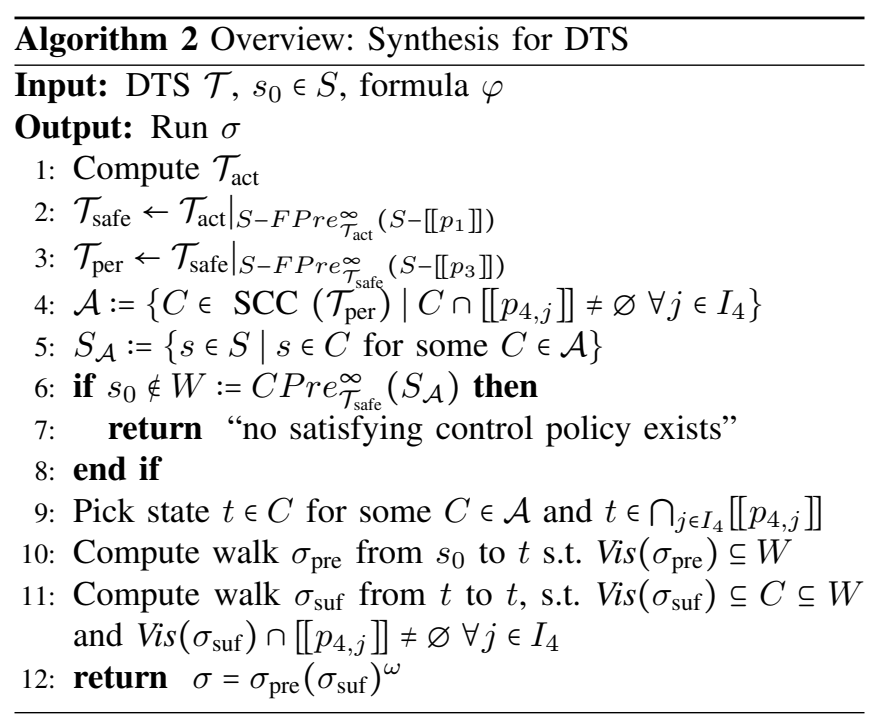

\section{NON-DETERMINISTIC TRANSITION SYSTEM}

We now discuss control policy construction for nondeterministic transition systems, which subsumes the development in Section IV. Our approach here differs primarily in the form of the control policy and how to determine the set of states that satisfy the recurrence formula, $\varphi_{\text {rec }}$.

We address formulas for $\varphi_{\text {act }}, \varphi_{\text {safe }}$, and $\varphi_{\text {per }}$ in a similar manner as Section IV because both the set FPre $e^{\infty}$ and the subgraph operation are already defined for non-deterministic transition systems.

Next, consider the recurrence specification $\varphi_{\text {rec }}=$ $\bigwedge_{j \in I_{4}} \square \diamond p_{4, j}$. An approach similar to the strongly connected component decomposition in Section IV could be used, but it is less efficient to compute due to the non-determinism. Büchi (and the more general parity) acceptance conditions have been extensively studied [10], [20].

Proposition 6. Algorithm 3 computes the winning set for $\varphi_{\text {rec }}$.

Proof: To satisfy the acceptance condition $\operatorname{Inf}(\sigma) \cap$ $\llbracket\left[p_{4, j}\right] \neq \varnothing$ for all $j \in I_{4}, F_{i} \subseteq C \operatorname{Pre} e_{\mathcal{T}}^{\infty}\left(F_{j}\right)$ must hold for all $i, j \in I_{4}$ for some $F_{j} \subseteq\left[\left[p_{4, j}\right]\right]$. Algorithm 3 initializes $F_{j}:=\left[\left[p_{4, j}\right]\right]$ for all $j \in I_{4}$ and iteratively removes states from $F_{i}$ that are not in $\operatorname{CPre} e_{\mathcal{T}}^{\infty}\left(F_{j}\right)$ for all $i, j \in I_{4}$. It terminates when $F_{i} \subseteq C \operatorname{Pre} e_{\mathcal{T}}^{\infty}\left(F_{j}\right)$ holds for all $i, j \in I_{4}$ or $F_{i}=\varnothing$ for some $i \in I_{4}$, i.e., the winning set is empty. At every iteration, it removes at least one state in $F=\bigcup_{j \in I_{4}} F_{j}$ or terminates.

The outer while loop runs at most $|F|$ iterations. During each iteration, the outer for loop computes $C \operatorname{Pr} e_{\mathcal{T}}^{\infty}$ and runs the inner for loop $\left|I_{4}\right|$ times. The inner for loop takes $O\left(\sum_{j \in I_{4}}\left|F_{j}\right|\right)$ time to perform the set intersections. Thus, the total complexity of Algorithm 3 is $O\left(|F|\left|I_{4}\right|\left(|S|+|R|+\sum_{j \in I_{4}}\left|F_{j}\right|\right)\right)$.

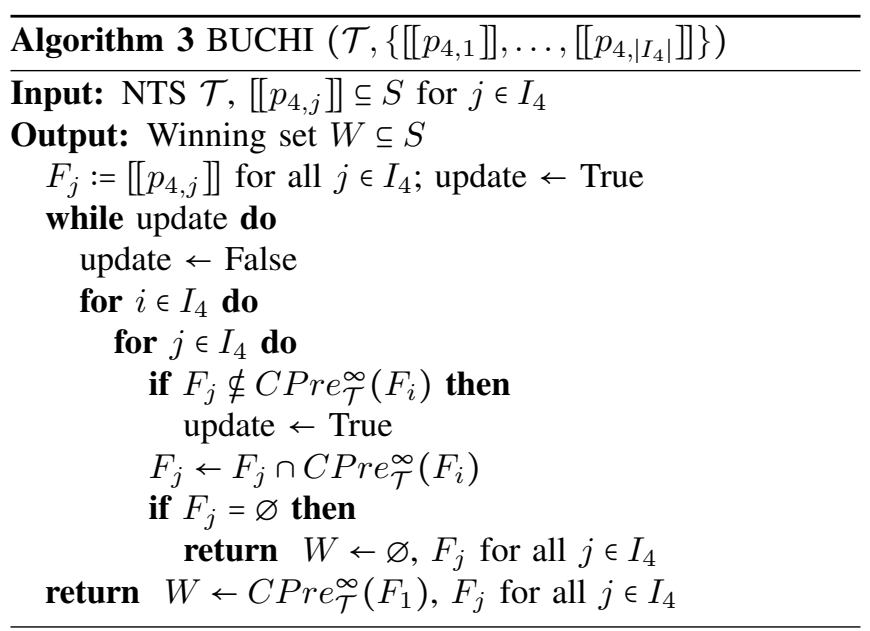

We now overview our approach for control policy synthesis for non-deterministic transition systems in Algorithm 4. Compute the set $W$ of states that are winning for $\varphi$ (lines 1$6)$. If the initial state $s_{0} \notin W$, then no control policy exists. If $s_{0} \in W$, compute the memoryless control policies $\mu_{j}$ induced from $V_{F_{j}, \mathcal{T}_{\text {safe }}}^{c}$ for all $j \in I_{4}$ (line 10, also see Algorithm 3). The finite-memory control policy $\mu$ is defined as follows by switching between memoryless policies depending on the current "target." Let $j \in I_{4}$ denote the current target set $F_{j}$. The system uses control policy $\mu_{j}$ until a state in $F_{j}$ is visited. Then, the system updates its "target" to $k=\left(j+1 \bmod \left|I_{4}\right|\right)+1$ and uses control policy $\mu_{k}$ until a state in $F_{k}$ is visited, and so on. The total complexity of the algorithm is $O(\alpha(|S|+|R|)+\beta)$, where $F=\bigcup_{j \in I_{4}}\left[\left[p_{4, j}\right]\right.$, $\alpha=\left|I_{2}\right|+\left|I_{4}\right||F|$, and $\beta=\left|I_{4}\right||F| \sum_{j \in I_{4}}\left|F_{j}\right|$.

\section{MARKov Decision Processes}

We now consider the Markov decision process (MDP) model. MDPs provide a general framework for modeling non-determinism (e.g., system actions) and probabilistic (e.g., environment actions) behaviors that are present in many real-world systems. We interpret the environment differently than in previous sections; it acts probabilistically through a transition probability function instead of nondeterministically. We sketch an approach for control policy synthesis for formulas of the form $\varphi=\varphi_{\text {safe }} \wedge \varphi_{\text {per }} \wedge \varphi_{\text {rec }}$ using techniques from probabilistic model checking [18]. This terse presentation will be extended in later publications. 


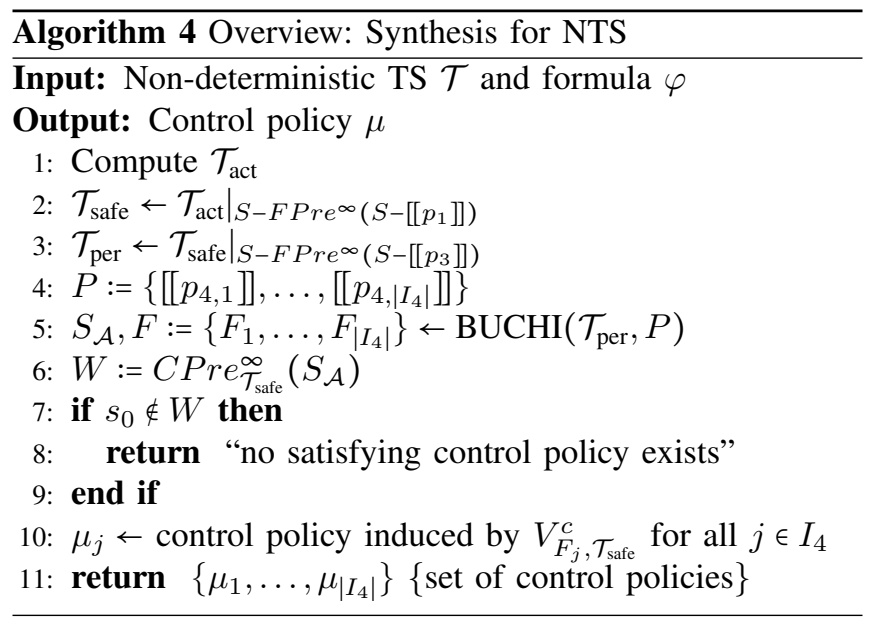

Definition 3. A (finite) labeled $M D P \mathcal{M}$ is the tuple $\mathcal{M}=\left(S, A, P, s_{0}, A P, L\right)$, consisting of a finite set of states $S$, a finite set of actions $A$, a transition probability function $P: S \times A \times S \rightarrow[0,1]$, an initial state $s_{0}$, a finite set of atomic propositions $A P$, and a labeling function $L: S \rightarrow$ $2^{A P}$. Let $A(s)$ denote the set of available actions at state $s$. Let $\sum_{s^{\prime} \in S} P\left(s, a, s^{\prime}\right)=1$ if $a \in A(s)$ and $P\left(s, a, s^{\prime}\right)=0$ otherwise. We assume, for notational convenience, that the available actions $A(s)$ are the same for every $s \in S$.

A run of the MDP is an infinite sequence of its states, $\sigma=s_{0} s_{1} s_{2} \ldots$ where $s_{i} \in S$ is the state of the system at index $i$ and $P\left(s_{i}, a, s_{i+1}\right)>0$ for some $a \in A\left(s_{i}\right)$. The set of runs of $\mathcal{M}$ with initial state $s$ induced by a control policy $\mu$ (as defined in Section II-A) is denoted by $\mathcal{M}^{\mu}(s)$. There is a probability measure over the runs in $\mathcal{M}^{\mu}(s)$ [18].

Given a run of $\mathcal{M}$, the syntax and semantics of LTL is identical to Section II-B. However, satisfaction for an MDP $\mathcal{M}$ under a control policy $\mu$ is now defined probabilistically [18]. Let $\mathbb{P}\left(M^{\mu}(s) \vDash \varphi\right)$ denote the expected satisfaction probability of LTL formula $\varphi$ by $\mathcal{M}^{\mu}(s)$.

Problem 2. Given an MDP $\mathcal{M}$ with initial state $s_{0}$ and an LTL formula $\varphi$, compute the control policy $\mu^{*}=$ $\arg \max _{\mu} \mathbb{P}\left(\mathcal{M}^{\mu}\left(s_{0}\right) \vDash \varphi\right)$, over all possible finite-memory, deterministic policies.

The value function at a state now has the interpretation as the maximum probability of the system satisfying the specification from that state. Let $B \subseteq S$ be a set from which the system can satisfy the specification almost surely. The value $V_{B, \mathcal{M}}(s)$ of a state $s \in S$ is the probability that the MDP $\mathcal{M}$ will reach set $B \subseteq S$ when using an optimal control policy starting from state $s \in S$.

We first compute the winning set $W \subseteq S$ for the LTL formula $\varphi=\varphi_{\text {safe }} \wedge \varphi_{\text {per }} \wedge \varphi_{\text {rec }}$. The probability of satisfying $\varphi$ is equivalent to the probability of reaching an accepting maximal end component [18]. Informally, accepting maximal end components are sets of states that the system can remain in forever and where the acceptance condition of $\varphi$ is satisfied almost surely. These sets can be computed in $O(|S||R|)$ time using graph search [18]. The winning set
TABLE I

COMPLEXITY OF CONTROL POLICY SYNTHESIS

\begin{tabular}{|l|l|l|l|}
\hline Language & DTS & NTS & MDP \\
\hline Frag. (1) & $O(|\varphi||\mathcal{T}|)$ & $O(\alpha|\mathcal{T}|+\beta)$ & $O(\operatorname{LP}(|\mathcal{T}|))$ \\
GR(1) & $O(|\varphi||S||R|)$ & $O(|\varphi||S||R|)$ & N/A \\
LTL & $O\left(|\mathcal{T}| 2^{(|\varphi|)}\right)$ & $O\left(|\mathcal{T}| 2^{2^{(|\varphi|)}}\right)$ & $O\left(\operatorname{LP}(|\mathcal{T}|) 2^{2^{(|\varphi|)}}\right)$ \\
\hline
\end{tabular}

$W \subseteq S$ is the union of all states that are in some accepting maximal end component.

\section{A. Reachability}

Once we have computed the winning set $W \subseteq S$ where the system can satisfy the specification $\varphi$ almost surely, we need to reach $W$ from the initial state $s_{0}$. Let $\mathcal{M}_{\text {safe }}$ be the sub-MDP (defined similarly to Section III-B, see [18]) where all states satisfy $\varphi_{\text {safe. }}$. The set $S_{1}=C \operatorname{Pr} e^{\infty}(W)$ contains all states that can satisfy $\varphi$ almost surely. Let $S_{r}$ be the set of states that have positive probability of reaching $W$, which can be computed by graph search [18]. The remaining states $S_{0}=S-\left(S_{1} \cup S_{r}\right)$ cannot reach $W$ and thus have zero probability of satisfying $\varphi$. Initialize $V_{B}^{c}(s)=1$ for all $s \in S_{1}$, $V_{B}^{c}(s)=0$ for all $s \in S_{0}$, and $V_{B}^{c}(s) \in(0,1)$ for all $s \in S_{r}$. It remains to compute the value function, i.e. the maximum probability of satisfying the specification, for each state in $S_{r}$. This computation boils down to a standard reachability problem that can be solved by linear programming or value iteration [18], [21].

\section{B. Control policy}

The control policy for maximizing the probability of satisfying the LTL formula $\varphi$ consists of two parts: a memoryless deterministic policy for reaching an accepting maximal end component, and a finite-memory deterministic policy for staying there. The former policy is computed from $V_{B, \mathcal{M}}^{c}$ and denoted $\mu_{\text {reach }}$. The latter policy is a finite-memory policy $\mu_{B}$ that selects actions to ensure that the system stays inside the accepting maximal end component forever and satisfies $\varphi$ by visiting every state infinitely often [18]. The control policy $\mu^{*}$ is $\mu^{*}=\mu_{\text {reach }}$ if $s \notin B$ and $\mu^{*}=\mu_{B}$ if $s \in B$.

\section{COMPLEXITY}

We summarize out complexity results and compare them with those for synthesis with LTL specifications and the GR(1) fragment of it [10]. In our analysis, we assume that set membership is determined in constant time with a hash function [22]. Let $|\mathcal{T}|=|S|+|R|$ denote the size of the system, $F=\bigcup_{j \in I_{4}}\left[\left[p_{4, j}\right]\right], \alpha=\left|I_{2}\right|+\left|I_{4}\right||F|$, and $\beta=\left|I_{4}\right||F| \sum_{j \in I_{4}}\left|F_{j}\right|$. Let $|\varphi|=\left|I_{2}\right|+\left|I_{4}\right|$ for fragment (1), $|\varphi|=m n$ for a GR(1) formula with $m$ assumptions and $n$ guarantees, and $|\varphi|$ be the length of formula $\varphi$ for LTL [18]. Let $\operatorname{LP}(|\mathcal{T}|)$ denote that the complexity is polynomial in $|\mathcal{T}|$, specifically that of solving a linear program. For typical motion planning specifications, $|F| \ll|S|, \sum_{j \in I_{4}}\left|F_{j}\right| \ll|S|$, and $|\varphi|$ is small. We use the non-symbolic complexity results for GR(1) in [10]. Results are summarized in Table I.

Remark 5. Fragment (1) is not handled well by standard approaches. Using the popular software ltl2ba [23], we 
created Büchi automaton for LTL formulas of the form $\varphi_{\text {act }}$. The automaton size and time to compute it both increased exponentially with the number of conjunctions in $\varphi_{\text {act }}$.

\section{EXTENSIONS}

We discuss two natural extensions to the fragment in formula (1). The first is includes guarantee and obligation properties, and the second includes disjunctions of formulas.

\section{A. Guarantee and obligation}

While guarantee and obligation, i.e., $\diamond p$ and $\square(p \Longrightarrow \diamond q)$ respectively (where $p$ and $q$ are propositional formulas), specifications are not explicitly included in (1), they can be incorporated by introducing new system variables, which exponentially increases the system size [7]. Even including conjunctions of guarantee formulas is NP-complete [24].

Another approach is to use the stricter specifications $\square \diamond$ $p$ for guarantee and $\square \neg p \vee \square \diamond q$ for obligation. The $\square \diamond$ formulas are part of the fragment in (1), and disjunctions can be included in some cases (see Section VIII-B). If the transition system is strongly connected, then these stricter formulas are feasible if and only if the original formulas are. Strong connectivity is a natural assumption in many robotics applications. For example, an autonomous car can typically drive around the block to revisit a location.

\section{B. Disjunctions of specifications}

We now consider an extension to specifications that are disjunctions of formulas of the form (1).

For a deterministic transition system, a control policy for a formula given by disjunctions of formulas of the form (1) can be computed by independently solving each individual subformula using the algorithms given earlier in this section.

Proposition 7. Let $\varphi=\varphi_{1} \vee \varphi_{2} \vee \ldots \vee \varphi_{n}$, where $\varphi_{i}$ is a formula of the form (1) for $i=1, \ldots, n$. Then, there exits a control policy $\mu$ such that $\mathcal{T}^{\mu}(s) \vDash \varphi$ if and only if there exists a control policy $\mu$ such that $\mathcal{T}^{\mu}(s) \vDash \varphi_{i}$ for some $i=1, \ldots, n$.

Proof: Sufficiency is obvious. For necessity, assume that there exists a control policy $\mu$ such that $\mathcal{T}^{\mu}(s)$ satisfies $\varphi$. The set $\mathcal{T}^{\mu}(s)$ contains a single run $\sigma$ since $\mathcal{T}$ is deterministic. Thus, $\sigma$ satisfies $\varphi_{i}$ for some $i=1, \ldots, n$.

For non-deterministic transition systems, necessity in Proposition 7 no longer holds because the non-determinism may be resolved in multiple ways and thus independently evaluating each subformula may not work.

Algorithm 5 is a sound, but not complete, procedure for synthesizing a control policy for a non-deterministic transition system with a specification given by disjunctions of formulas of the form (1). Arbitrary disjunctions of this form are intractable to solve exactly, as this extension subsumes Rabin games which are NP-complete [25].

Algorithm 5 computes winning sets $W_{i} \subseteq S$ for each subformula $\varphi_{i}$ and checks if the initial state can reach their union $\mathcal{W}:=\bigcup_{i=1}^{n} W_{i}$. The control policy $\mu_{\text {reach }}$ is used until a state $s \in W_{i}$ is reached for some $i$, after which $\mu_{i}$ is used.
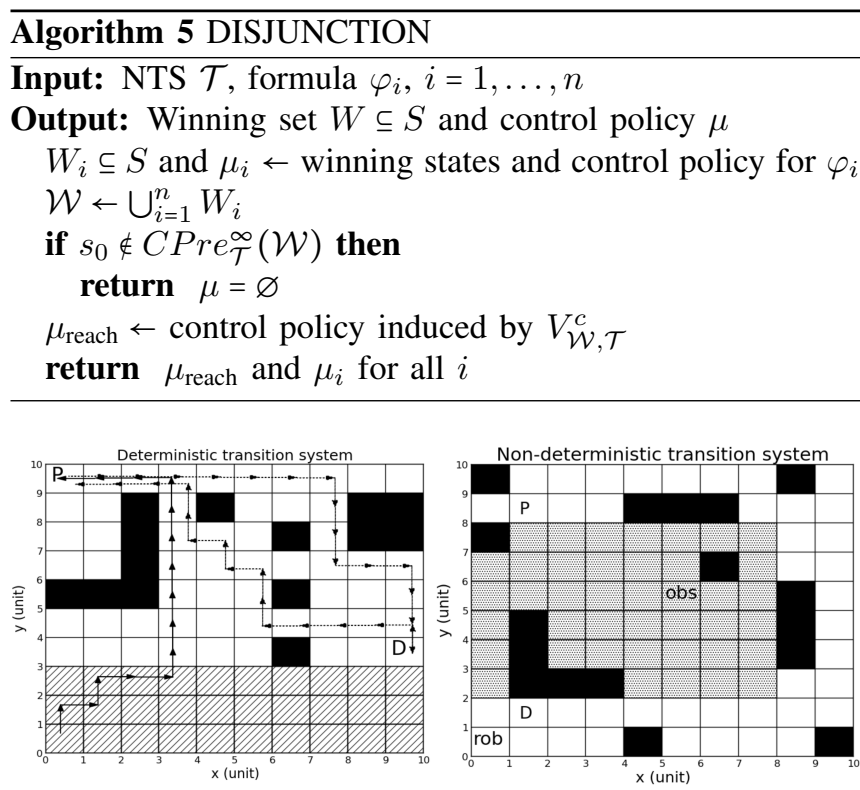

Fig. 2. Left: Diagram of a $10 \times 10$ deterministic grid. Only white cells are labeled 'stockroom.' Right: Diagram of a 10 x 10 non-deterministic grid with a dynamic obstacle (obs) that moves within the shaded region.

\section{EXAMPLES}

The following examples demonstrate the techniques developed in Sections IV and V for tasks motivated by robot motion planning in a planar environment (see Figure 2). We defer an example for Section VI due to space limitations. Computations were done in Python on a dual-core Linux desktop with $2 \mathrm{~GB}$ of memory. All computation times were averaged over five randomly generated problem instances. Including the transition system construction roughly doubled the computation time in these examples.

\section{A. Deterministic transition system}

Consider a gridworld where a robot occupies a single cell at a time and can choose to either remain in its current cell or move to one of four adjacent cells at each step. We consider square grids with static obstacle densities of 15 percent. The set of atomic propositions is $A P=\{$ pickup, dropoff, storeroom, obs $\}$. The robot's task is to eventually remain in the stockroom while repeatedly visiting a pickup and a dropoff location. The robot must never collide with a static obstacle. This task is formalized by the LTL formula $\varphi=\diamond \square$ stockroom $\wedge \square \diamond$ pickup $\wedge \square \diamond$ dropoff $\wedge \square \neg$ obs, which is in fragment (1).

Results are shown in Figure 3. A corresponding nondeterministic Büchi automaton for $\varphi$ has four states [23]. Thus, the standard automata-based approach for LTL would do similar graph search computations on a graph four times larger than the transition system.

\section{B. Non-deterministic transition system}

We now consider a similar setup as in Section IX-A, but with a dynamically moving obstacle. The state of the system is the product of the robot's location and the obstacle's location, both of which can move as previously described for 


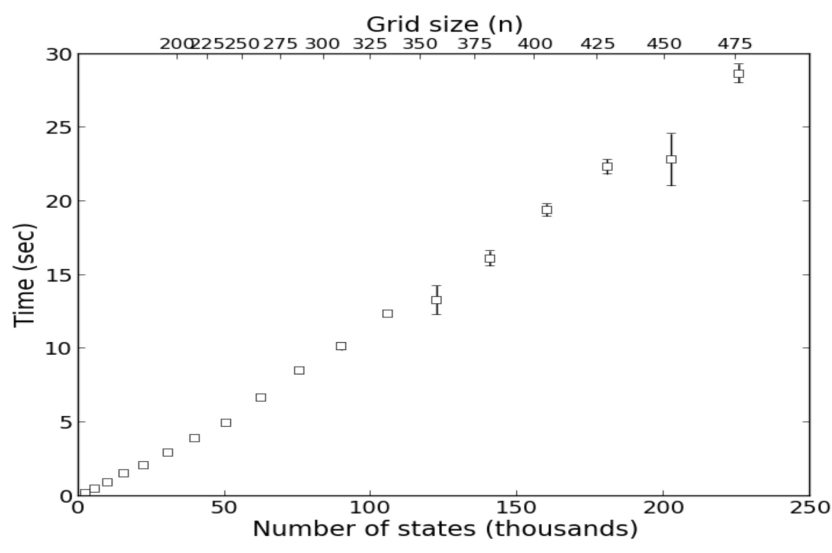

Fig. 3. Control policy synthesis times for deterministic grids.

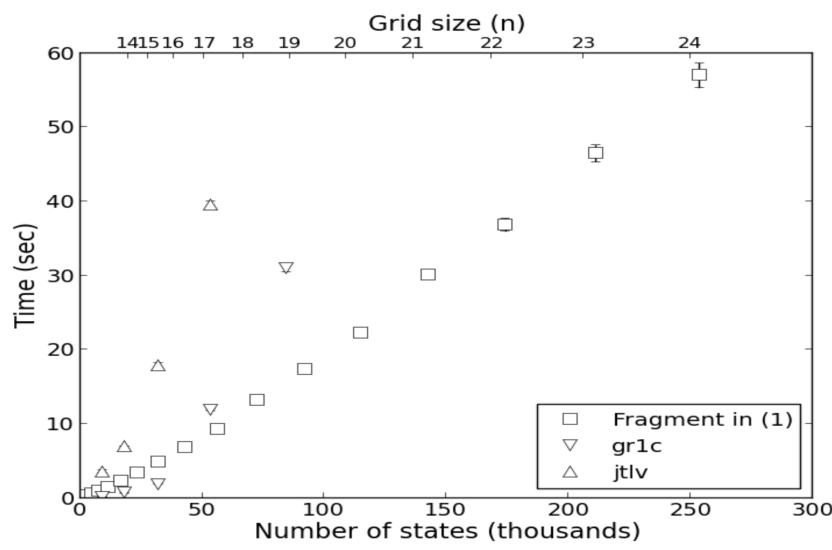

Fig. 4. Control policy synthesis times for non-deterministic grids.

the robot. The robot selects an action and then the obstacle non-deterministically moves. The robot's task is to repeatedly visit a pickup and a dropoff location while never colliding with an obstacle. This task is formalized by the LTL formula $\varphi=\square \diamond$ pickup $\wedge \square \diamond$ dropoff $\wedge \square \neg$ obs, which is in both fragment (1) and GR(1) [10].

Results are shown in Figure 4. We compare our algorithm to two implementations (jtlv and gr1c as used in [26]) of the GR(1) synthesis method from [10]. Our algorithms scale significantly better; neither the jtlv or gr1c implementation was able to solve a problem with over 100 thousand states.

\section{Conclusions}

We presented a framework for control policy synthesis for both non-deterministic transition systems and Markov decision processes that are subject to temporal logic task specifications. Our approach for control policy synthesis is straightforward and efficient, both theoretically and according to our preliminary experimental results. It offers a promising alternative to the commonly used GR(1) specifications as it can express many relevant tasks for multiple system models.

Future work will extend the synthesis algorithms here to create optimal control policies for systems with cost functions. Incremental synthesis methods for computing the reachable sets also appear promising. Finally, detailed experimental analysis is needed to compare practical performance to GR(1) and automata-based methods.

\section{ACKNOWLEDGEMENTS}

The authors would like to thank Scott Livingston, Matanya Horowitz, and the anonymous reviewers for helpful input. This work was supported by a NDSEG fellowship, the Boeing Corporation, and AFOSR award FA9550-12-1-0302.

\section{REFERENCES}

[1] C. Courcoubetis and M. Yannakakis, "The complexity of probabilistic verification," Journal of the ACM, vol. 42, pp. 857-907, 1995.

[2] A. Pnueli and R. Rosner, "On the synthesis of a reactive module," in Proc. Symp. on Princp. of Prog. Lang., 1989, pp. 179-190.

[3] S. Karaman and E. Frazzoli, "Sampling-based motion planning with deterministic $\mu$-calculus specifications," in Proc. of IEEE Conf. on Decision and Control, 2009.

[4] E. Plaku, L. E. Kavraki, and M. Y. Vardi, "Motion planning with dynamics by a synergistic combination of layers of planning," IEEE Trans. on Robotics, vol. 26, pp. 469-482, 2010.

[5] M. Kloetzer and C. Belta, "A fully automated framework for control of linear systems from temporal logic specifications," IEEE Trans. on Automatic Control, vol. 53, no. 1, pp. 287-297, 2008.

[6] H. Kress-Gazit, G. E. Fainekos, and G. J. Pappas, "Temporal logicbased reactive mission and motion planning," IEEE Trans. on Robotics, vol. 25 , pp. 1370-1381, 2009.

[7] T. Wongpiromsarn, U. Topcu, and R. M. Murray, "Receding horizon temporal logic planning," IEEE Trans. on Automatic Control, 2012.

[8] X. C. Ding, S. L. Smith, C. Belta, and D. Rus, "LTL control in uncertain environments with probabilistic satisfaction guarantees," in Proc. of 18th IFAC World Congress, 2011.

[9] M. Lahijanian, S. B. Andersson, and C. Belta, "Temporal logic motion planning and control with probabilistic satisfaction guarantees," IEEE Trans. on Robotics, vol. 28, pp. 396-409, 2012.

[10] R. Bloem, B. Jobstmann, N. Piterman, A. Pnueli, and Y. Sa'ar, "Synthesis of Reactive(1) designs," Journal of Computer and System Sciences, vol. 78, pp. 911-938, 2012.

[11] R. Alur and S. La Torre, "Deterministic generators and games for LTL fragments," ACM Trans. Comput. Logic, vol. 5, no. 1, pp. 1-25, 2004.

[12] O. Maler, A. Pnueli, and J. Sifakis, "On the synthesis of discrete controllers for timed systems," in STACS 95. Springer, 1995, vol. 900, pp. 229-242.

[13] R. Ehlers, "Generalized Rabin(1) synthesis with applications to robust system synthesis," in NASA Formal Methods. Springer, 2011.

[14] C. Belta and L. C. G. J. M. Habets, "Controlling of a class of nonlinear systems on rectangles," IEEE Trans. on Automatic Control, vol. 51, pp. 1749-1759, 2006.

[15] L. Habets, P. J. Collins, and J. H. van Schuppen, "Reachability and control synthesis for piecewise-affine hybrid systems on simplices," IEEE Trans. on Automatic Control, vol. 51, pp. 938-948, 2006.

[16] S. LaValle and J. J. Kuffner, "Randomized kinodynamic planning," Int. Journal of Robotics Research, vol. 20, pp. 378-400, 2001.

[17] L. E. Kavraki, P. Svestka, J. C. Latombe, and M. H. Overmars, "Probabilistic roadmaps for path planning in high-dimensional configuration spaces," IEEE Trans. Robot. Autom., vol. 12, pp. 566-580, 1996.

[18] C. Baier and J.-P. Katoen, Principles of Model Checking. MIT Press, 2008.

[19] E. A. Emerson, "Handbook of theoretical computer science (vol. B)," in Temporal and modal logic, J. van Leeuwen, Ed. MIT Press, 1990, ch. Temporal and modal logic, pp. 995-1072.

[20] E. Gradel, W. Thomas, and T. Wilke, Eds., Automata, Logics, and Infinite Games: A Guide to Current Research. Springer-Verlag New York, Inc., 2002.

[21] D. P. Bertsekas, Dynamic Programming and Optimal Control (Vol. I and II). Athena Scientific, 2001.

[22] T. H. Cormen, C. E. Leiserson, R. L. Rivest, and C. Stein, Introduction to Algorithms: 2nd ed. MIT Press, 2001.

[23] P. Gastin and D. Oddoux, "Fast LTL to Büchi automata translation," in Proc. of the 13th Int. Conf. on Computer Aided Verification, 2001.

[24] A. Sistla and E. Clarke, "The complexity of propositional linear temporal logics," Journal of the ACM, vol. 32, pp. 733-749, 1985.

[25] E. Emerson and C. Jutla, "The complexity of tree automata and logic of programs," in In 29th FOCS, 1988.

[26] T. Wongpiromsarn, U. Topcu, N. Ozay, H. Xu, and R. M. Murray, "TuLiP: A software toolbox for receding horizon temporal logic planning," in Proc. of Int. Conf. on Hybrid Systems: Computation and Control, 2011, http://tulip-control.sf.net. 